\title{
O AJUSTAMENTO DE CRIANÇAS COM DOENÇA CRÔNICA
}

\author{
THE ADJUSTMENT OF CHILDREN WITH CHRONIC ILLNESS \\ EL AJUSTE DE LOS NIÑOS CON ENFERMEDADES CRÓNICAS
}

\author{
Joana Matias Antão* \\ Maria Gabriela Correia de Castro Portugal ${ }^{*}$ \\ Carlos Fernandes da Silva**
}

\begin{abstract}
RESUMO
Este trabalho procura averiguar o impacte das doenças crônicas no ajustamento psicológico das crianças, tendo em conta diferentes tipos de doenças, suas características e a percepção dos pais. A amostra é constituída por 176 crianças, dos 3 aos 10 anos, distribuídas por quatro grupos: crianças com asma, com câncer, com patologia uronefrológica e sem doença. A recolha de dados ocorreu nas salas de espera de dois hospitais portugueses. Os instrumentos utilizados foram a escala de observação do brincar (POS), itens do revised illness perception questionnaire (IPQ-R) e o questionário de capacidades e de dificuldades (SDQ). A análise dos resultados permitiu perceber que não existe uma relação linear entre o ajustamento psicológico e a presença de uma doença crônica, e que a avaliação do ajustamento da criança pela observação direta do brincar nem sempre é coincidente com a perspectiva dos pais acerca desse ajustamento.
\end{abstract}

Palavras-chave: Doença crônica. Ajustamento. Brincar. Infância.

\section{ABSTRACT}

This study focus on the impact of chronic disease over the children's psychological adjustment, based on the different types of diseases, their characteristics and parents' perception. The sample is composed of 176 children, aged between 3 and 10 years, distributed in four groups: children with asthma, cancer, uro-nephrological pathology and free from any disease. The data were collected in the waiting rooms of two Portuguese hospitals. The Play Observation Scale (POS), items from the Revised Illness Perception Questionnaire (IPQ-R) and the Strengths and Difficulties Questionnaire (SDQ) were the tools applied in the study. The analysis of the results revealed that a linear relationship between the psychological adjustment of children and the presence of a chronic disease does not exist. In addition, the evaluation of the child's adjustment through the direct observation of

Texto recebido em 18 de janeiro de 2016 e aprovado para publicação em 2 de setembro de 2016.

Universidade de Aveiro, Portugal. E-mail: joana.antao@gmail.com.

"Universidade de Aveiro, Portugal. E-mail: gabriela.portugal@ua.pt.

${ }^{* *}$ Universidade de Aveiro, Portugal. E-mail: csilva@ua.pt. 
ludic activities does not always match the parents' perspective regarding that adjustment.

Keywords: Chronic disease. Adjustment. Ludic activities. Childhood.

\section{RESUMEN}

Este trabajo trata de determinar el impacto de las enfermedades crónicas en el ajuste psicológico de los niños, teniendo en cuenta los diferentes tipos de enfermedades, sus características y la percepción de los padres acerca de ellos. La muestra se compone de 176 niños, de 3 a 10 años, divididos en cuatro grupos: los niños con asma, cáncer, patologías uronefrológicas y sin enfermedad. La recolección de datos ocurrió en las salas de espera de dos hospitales portugueses. Los instrumentos utilizados fueron la Play Observation Scale (POS), lo Revised Illness Perception Questionnaire (IPQ-R) y lo Strengths and Difficulties Questionnaire (SDQ). Los resultados nos permiten percibir que existe una relación lineal entre el ajuste psicológico y la presencia de una enfermedad crónica y que la evaluación de la adaptación de los niños a través de la observación directa del juego no siempre es coincidente con la visión de los padres sobre este ajuste.

Palabras clave: Enfermedad crónica. Ajuste. Jugar. Infancia.

\section{INTRODUÇÃO}

definição de doença crônica nem sempre é consensual, devido ao grande
leque de doenças que podem ser inseridas nessa categoria, podendo ser
considerado um conceito "flexível e em evolução" (Barros, 2003, p. 137). Pless e Pinkerton (1975) identificam critérios objetivos de caracterização dessas doenças, nomeadamente terem uma duração superior a três meses (no período de um ano) ou a necessidade de uma hospitalização de, pelo menos, um mês. Existindo diversas definições de doença crônica, todas parecem concordar no fato de essa condição ser prolongada e poder ter progressōes muito diferentes, desde uma esperança média de vida normal até à morte precoce (Bradford, 1997). Segundo a Organização Mundial de Saúde (OMS), essas doenças são a principal causa de morte no mundo. Estima-se que cerca de 10 a 20 por cento da população infantil tenha alguma doença crônica (Wallander \& Thompson, 1995; Weiland, Pless, \& Roghmann, 1992), sendo que apenas 1 a 2\% apresentam condições mais severas (Gortmaker \& Sappenfield, 1984 apud Wallander \& Thompson, 1995).

Sendo tão extenso o leque de doenças crônicas, tem sido discutida sua classificação, podendo ser encontradas duas visões distintas em relação a este 
assunto: visão idiossincrática e visão não categorial (Eiser, 1993). A visão idiossincrática baseia-se na premissa de que as doenças são todas diferentes e, por isso, suas consequências são também diversas (Eiser, 1993), defendendo o agrupamento de doenças crônicas especificas (Thompson \& Gustafson, 1996). No entanto, Pless e Pinkerton (1975) chamam a atenção para o fato de existirem problemas que são comuns a todas as doenças crônicas. Dessa forma, é cada vez maior o número de investigadores que defende uma visão não categorial, considerando que as tentativas de encontrar diferenças entre as doenças apenas têm servido para ocultar as semelhanças que existem entre si (Bradford, 1997). De acordo com essa visão, existem características comuns a diferentes doenças, como a visibilidade, a natureza e curso da doença, o tipo de tratamento, entre outras. A adaptação da criança à doença é vista em razão dessas dimensões e não do tipo de doença (Thompson \& Gustafson, 1996; Wallander \& Varni, 1998). Thompson e Gustafson (1996) consideram que existem vantagens e desvantagens nas duas abordagens, optando por uma terceira visão: parcial ou modificada. De acordo com essa perspectiva, existem simultaneamente questóes que são específicas de determinadas doenças e processos que são genéricos e comuns a várias.

Este estudo (Antão, 2013) pretende analisar efeitos das doenças crônicas no ajustamento psicológico das crianças. Embora o conceito de ajustamento seja referido com bastante frequência, este é muito difícil de definir e de objetivar (Pless \& Pinkerton, 1975). Wallander e Thompson (1995) relacionam esse conceito com os de adaptação e saúde mental, sendo que ambos se referem ao funcionamento emocional e social do indivíduo. Segundo esses autores, para definir ajustamento é fundamental assumir uma perspectiva normativodesenvolvimental, segundo a qual um ajustamento positivo na infância, associado a comportamentos adequados, normativos e saudáveis, está associado a um funcionamento positivo na idade adulta (Wallander \& Thompson, 1995). $\mathrm{Na}$ área da doença crônica, é necessário ter particular atenção à definição e aplicação desse conceito, sendo importante fazer a distinção entre o conceito de ajustamento como adaptação à doença e o conceito de ajustamento em seu sentido mais lato, ou seja, como medida do funcionamento da criança no seu dia a dia (Eiser, 1993; Pless \& Pinkerton, 1975). Dados os objetivos deste estudo, centrar-nos-emos na definição de ajustamento em seu sentido mais alargado.

\subsection{Objetivos}

Este estudo tem os seguintes objetivos: averiguar a existência de diferenças no ajustamento psicológico de crianças com e sem doença crônica e com doenças 
crônicas diferentes (patologia uronefrológica, asma e câncer); investigar o impacte das características da doença no ajustamento psicológico da criança; investigar a relação entre a percepção dos pais acerca da doença e o ajustamento psicológico da criança.

\section{MÉTODO}

\subsection{Participantes}

A amostra é constituída por 176 crianças, dos 3 aos 10 anos $(\underline{M}=5,90, \underline{S D}=$ 2.221), sendo 74 do sexo masculino e 102 do sexo feminino, que frequentavam, à data do estudo, o serviço de Consulta Externa de Pediatria do Hospital Infante D. Pedro (Aveiro) e do Hospital Pediátrico de Coimbra.

A amostra é de conveniência, uma vez que, por motivos de operacionalização da pesquisa, apenas foram observadas as crianças que frequentavam esses dois hospitais. Para além disso, apenas integraram o estudo as crianças cujos pais concederam autorização para a participação, mediante consentimento informado, aceitando a cooperação na investigação.

Relativamente à distribuição da amostra de acordo com o diagnóstico, verificase que 32 crianças não têm doença crônica, 47 têm câncer, 62 têm asma e 35 têm alguma patologia uronefrológica.

\subsection{Procedimentos}

Nesta investigação, foi respeitado o código de ética da investigação, sendo salvaguardadas todas as implicações de natureza ética dos participantes envolvidos.

A recolha de dados foi realizada em dois hospitais portugueses: o Hospital Infante D. Pedro e o Hospital Pediátrico de Coimbra. Essa recolha assumiu dois formatos: a aplicação de questionários aos pais das crianças e a observação das crianças nas salas de espera. A investigadora esteve presente 24 vezes, durante cerca de $2 \mathrm{~h} 30 \mathrm{~min}$, em cada consulta externa: oncologia médica, patologia alérgica respiratória e patologia uronefrológica

De cada vez que a investigadora se dirigiu a uma das salas de espera, obteve o consentimento informado dos pais/cuidadores das várias crianças que passavam por aquele espaço, explicando-lhes o intuito desta investigação e informandoos que os dados obtidos eram anônimos e confidenciais. Depois de entregue o questionário aos cuidadores, a investigadora iniciava a observação das crianças, seguindo uma ordem de observação definida aleatoriamente. Essa ordem era 
cumprida independentemente da atividade que cada criança estivesse realizando, para não enviesar os resultados.

\subsection{Instrumentos}

\subsubsection{Observação das crianças}

A observação do brincar das crianças teve lugar em contexto hospitalar, com apoio da Play Observation Scale (POS) (Rubin, 2001). Essa escala é de fácil e rápida aplicação: consiste em observar cada criança durante intervalos de dez segundos e registar, num quadro de observação, qual o comportamento mais presente durante esse período.

Esse quadro de observação baseia-se em dois tipos de classificações do brincar: de acordo com a dimensão social (Parten, 1932) e com a dimensão cognitiva (Piaget, 2010). Dessa forma, essa escala é dividida em comportamentos de brincar e de não brincar. Essa escala apresenta níveis elevados de fiabilidade interobservador: entre os $80 \%$ e os $90 \%$.

\subsubsection{Questionário preenchido pelo cuidador}

Foi construído um questionário, com respostas abertas e fechadas, a ser preenchido pelo cuidador que se encontrava acompanhando a criança ao hospital.

Com o objetivo de compreender os contextos de vida da criança, foi construído um questionário sociodemográfico composto por duas partes. A primeira diz respeito à identificação dos pais (sexo e idade), à filiação (número de filhos) e à saúde dos restantes filhos. A segunda parte desse questionário centra-se na criança-alvo, procurando ter acesso a seus dados de identificação gerais bem como alguns dados objetivos referentes a seu estado de saúde e à severidade da doença.

De forma, observando qual a percepção que os pais tinham acerca da doença de seu filho, foram adaptados alguns itens do Revised Illness Perception Questionnaire (IPQ-R). Esse questionário encontra-se adaptado e validado para português (Santos, Pais-Ribeiro, \& Lopes, 2003), destinando-se a avaliar a representação que os próprios doentes têm de sua enfermidade. O IPQ-R é composto por três subescalas. Para este estudo, apenas foi usada a segunda subescala, sendo apenas selecionado um item referente a cada uma das categorias (Quadro 1). Para cada um dos itens, as respostas orientam-se numa escala de tipo Likert de cinco pontos em que 1 - "discorda plenamente" e 5 - "concorda plenamente". Os itens referentes ao controle pessoal (pais) e duração são itens de cotação 
inversa.

\begin{tabular}{|c|c|}
\hline & Itens selecionados \\
\hline Duração & A doença do meu filho vai durar muito tempo \\
\hline Consequências & $\begin{array}{c}\text { A doença do meu filho tem grandes consequências na } \\
\text { vida dele }\end{array}$ \\
\hline Controle pessoal (criança) & $\begin{array}{l}\text { O que o meu filho faz pode determinar se a sua doença } \\
\text { melhora ou piora }\end{array}$ \\
\hline Controle pessoal (pais) & $\begin{array}{c}\text { As minhas ações não terão qualquer efeito no resultado } \\
\text { da doença do meu filho }\end{array}$ \\
\hline Controle de tratamento & $\begin{array}{l}\text { O tratamento será eficaz na cura da doença do meu } \\
\text { filho }\end{array}$ \\
\hline Coerência da doença & Não compreendo a doença do meu filho \\
\hline Duração (cíclica) & $\begin{array}{c}\text { Passo por fases em que a doença do meu filho melhora } \\
\text { ou piora }\end{array}$ \\
\hline Representação emocional & A doença do meu filho não me preocupa \\
\hline
\end{tabular}

Fonte: elaborado pelos autores.

O Strengths and Difficulties Questionnaire (SDQ) é um questionário breve de avaliação comportamental de crianças e adolescentes entre os 3 e os 16 anos. Todas as versões são compostas por três partes: 25 itens sobre atributos psicológicos, suplemento de impacte e questóes de follow up. Neste estudo, apenas será usada a primeira parte, sendo o questionário preenchido pelos pais.

Os 25 itens sobre atributos psicológicos reportam-se a atributos positivos e negativos, encontrando-se divididos em cinco escalas: problemas de comportamento, hiperatividade, sintomas emocionais, problemas de relacionamento com os colegas; e comportamento pró-social. A soma das quatro primeiras escalas é referente ao total das dificuldades. Cada um desses itens é respondido numa escala de Likert de três pontos, sendo que (0) não é verdade, (1) é um pouco verdade (2) é muito verdade. Neste estudo, para cada questão, foi ainda pedido aos pais que assinalassem se cada comportamento estava ou não associado à doença do seu filho.

\section{RESULTADOS}

\subsection{Objetivo 1: explorar o ajustamento psicológico de crianças com doenças crônicas diferentes}

Um dos objetivos deste estudo é perceber se crianças com doenças crônicas distintas se diferenciam em termos de ajustamento psicológico, dependendo do tipo de doença e, ou, se apresentam diferenças em comparação com crianças 
sem doença crônica. Para avaliação do ajustamento psicológico das crianças, foi aplicado aos pais o Questionário de Capacidades e de Dificuldades (SDQ - Por), e o brincar das crianças foi observado na sala de espera com recurso à Escala de Observação do Brincar (POS).

\subsubsection{Questionário de Capacidades e de Dificuldades (SDQ)}

A comparação entre os vários grupos de crianças para cada uma das subescalas permite perceber que apenas foram encontradas diferenças estatisticamente significativas, $\mathrm{F}(3,157)=4.167, \mathrm{p}<.01$, na subescala "Sintomas emocionais". A comparação das médias permite perceber que existem diferenças entre o grupo de crianças com câncer e os restantes grupos, sendo o grupo de crianças com câncer o que apresenta valores mais elevados nesta subescala.

Em relação à atribuição do comportamento à doença por grupo foi possível constatar a existência de diferenças estatisticamente significativas em relação às questóes: "Queixa-se frequentemente de dores de cabeça, dores de barriga ou vómitos", $\chi^{2}(2,95)=31.663, \mathrm{p}<.01$; "Enerva-se muito facilmente e faz muitas birras", $\chi^{2}(2,95)=32.549, \mathrm{p}<.01$; "Tem muitas preocupaçôes, parece sempre preocupado/a", $\chi^{2}(2,95)=7.943, \mathrm{p}<.05$; "Gosta de ajudar se alguém está magoado, aborrecido ou doente", $\chi^{2}(2,95)=8.289, \mathrm{p}<.05$; "Anda muitas vezes triste, desanimado/a ou choroso/a", $\chi^{2}(2,94)=6.049, \mathrm{p}<.05$; "Em situações novas é receoso/a, muito agarrado/a e pouco seguro/a", $\chi^{2}(2,94)=12.057$, p < .01 ; "Mente frequentemente ou engana", $\chi^{2}(2,94)=11.310, \mathrm{p}<.01$; e "Tem muitos medos, assusta-se com facilidade", $\chi^{2}(2,94)=20.648, p<.01$. Em todas essas questões, são os pais das crianças com câncer os que mais atribuem a causa desses comportamentos à doença.

\subsubsection{Escala de Observação do Brincar (POS)}

Relativamente ao brincar, é possível observar que apenas foram encontradas diferenças estatisticamente significativas no que concerne ao brincar em grupo, $\mathrm{F}(3,172)=3.321, \mathrm{p}<.05$, entre o grupo de crianças sem doença e os restantes grupos, sendo as crianças sem doença as que apresentam menos comportamentos de brincadeira em grupo com os pares.

Foi ainda averiguada a existência de diferenças entre os grupos no que se refere ao tipo de brinquedos selecionados, não existindo diferenças estatisticamente significativas entre os grupos para os brinquedos gerais, $F(3,171)=0.752$, ns, nem para os brinquedos médicos, $F(3,172)=1.556$, ns. No entanto foi encontrada uma diferença marginalmente significativa nos brinquedos médicos 
$(\mathrm{p}=0.52)$, sendo que as crianças com câncer selecionam mais esses brinquedos do que as crianças sem nenhuma doença.

\subsection{Objetivo 2: investigar o impacte das características da doença no ajustamento da criança}

Este estudo pretende também averiguar o impacto das características da doença no ajustamento psicológico da criança.

\subsubsection{Questionário de Capacidades e Dificuldades (SDQ)}

Relativamente ao SDQ, é possível constatar que não foram encontradas diferenças estatisticamente significativas em relação ao SDQ total, subescala de problemas de comportamento, à subescala hiperatividade, à subescala problemas de relacionamento com os colegas e à subescala de comportamento pró-social. No que se refere à subescala de sintomas emocionais, é possível observar uma correlação significativa fraca com a variável tempo de doença, $\mathrm{r}=-.282, \mathrm{p}<.01$, sendo que quanto maior o tempo de doença, menor o valor na subescala de sintomas emocionais. Também foi encontrada uma correlação fraca no que se refere à periodicidade com que a criança vai ao hospital, $\mathrm{r}=-.279, \mathrm{p}<.01$, sendo que quanto maior a periodicidade com que a criança vai ao hospital, maior o valor nesta subescala. Foram ainda encontradas diferenças estatisticamente significativas no que se refere à impossibilidade de realizar alguma brincadeira, $\mathrm{t}$ $(131)=-2.662, \mathrm{p}<.01$, sendo que as crianças que não podem realizar algumas brincadeiras são as que apresentam valores mais elevados nesta subescala. Relativamente à medicação, encontraram-se diferenças estatisticamente significativas entre as crianças que tomam apenas comprimidos e as que estão em tratamento de quimioterapia, sendo que são estas últimas as que apresentam valores mais elevados nesta subescala.

\subsubsection{Escala de Observação do Brincar (POS)}

No que se refere à variável "brincar", "brincar solitário" e "brincar paralelo", foi possível constatar que não existem diferenças estatisticamente significativas em relação a nenhuma das variáveis relativas à saúde objetiva da criança.

Foram encontradas diferenças estatisticamente significativas em relação à impossibilidade de realização de alguma brincadeira para o "brincar em grupo", $\mathrm{t}(110,742)=2.213, \mathrm{p}<.05$, sendo que as crianças que não são impedidas de realizar nenhuma brincadeira são as que apresentam mais comportamentos de brincar em grupo. 


\subsection{Objetivo 3: investigar a relação entre a percepção dos pais acerca da doença e o ajustamento da criança.}

Este estudo pretende também averiguar a existência de diferenças ao nível do ajustamento psicológico tendo em conta a saúde subjetiva da criança. A saúde subjetiva da criança foi medida por alguns itens do IPQ-R.

\subsubsection{Questionário de Capacidades e Dificuldades (SDQ)}

Em relação ao SDQ total, subescala de problemas de comportamento, na subescala problemas de relacionamento com os colegas e na subescala de comportamento pró-social, não foram encontradas diferenças estatisticamente significativas em nenhuma das questôes do IPQ-R.

Relativamente à subescala de sintomas emocionais, encontrou-se uma correlação fraca entre esta subescala e a questão "A doença do meu filho tem grandes consequências na vida dele", $\mathrm{r}=.199, \mathrm{p}<.05$, sendo que quanto maiores as consequências percebidas pelos pais, maior o valor na escala de sintomas emocionais. $\mathrm{O}$ mesmo se dá relativamente à questão "O que o meu filho faz pode determinar se a sua doença melhora ou piora", sendo encontrada uma correlação fraca $\mathrm{r}=.209, \mathrm{p}<.05$, sendo que quanto maior a percepção de controle por parte do filho, maior o valor na escala de sintomas emocionais. Relativamente à questão "Não compreendo a doença do meu filho", foi também encontrada uma correlação significativa fraca, $\mathrm{r}=.245, \mathrm{p}<.01$, sendo que quanto menor a coerência atribuída à doença, maior o valor na escala de sintomas emocionais.

Relativamente à subescala hiperatividade, foi encontrada uma correlação baixa estatisticamente significativa na questão "Passo por fases em que a doença do meu filho melhora ou piora", $\mathrm{r}=.235, \mathrm{p}<.01$, sendo que quanto maior a percepção de uma duração cíclica da doença, maior o valor na subescala de hiperatividade.

\subsubsection{Escala de Observação do Brincar - POS}

Quanto ao brincar, foi encontrada uma correlação fraca estatisticamente significativa, $\mathrm{r}=.212, \mathrm{p}<.01$ com a questão "O tratamento será eficaz na cura da doença do meu filho". Dessa forma, quando maior a percepção dos pais acerca da eficácia do tratamento, maior a frequência de comportamentos de brincar.

Não foram encontradas correlações significativas em relação a nenhuma categoria do brincar social. 


\subsubsection{Saúde objetiva e saúde subjetiva}

De forma a perceber qual das variáveis teve um maior impacto na subescala sintomas emocionais, foi realizada uma regressão linear múltipla passo a passo, tendo esta subescala como variável dependente e, como independentes, variáveis relacionadas com as características da doença (tempo de doença, periodicidade, impossibilidade de realizar alguma brincadeira, internamentos e faltas à escola) e com a percepção dos pais (itens do IPQ-R). Pela regressão linear múltipla passo a passo, obtiveram-se três modelos. Destes, o terceiro modelo tem um $\mathrm{R}^{2}=.160$ e um $\mathrm{R}^{2}$ (ajustado) = .138. Foi possível concluir que as variáveis que mais se relacionam com a subescala sintomas emocionais são a periodicidade com que a criança se desloca ao hospital (saúde objetiva) e dois itens do IPQ-R relacionados com a compreensão da doença e com a percepção de controlo da doença por parte da criança (saúde subjetiva). Dessa forma, quanto maior a periodicidade com que a criança vai ao hospital e a percepção de controle por parte do filho, e quanto menor a coerência atribuída a doença, maior o valor nesta subescala.

\section{DISCUSSÃO}

Pela comparação entre os três grupos de crianças, é possível constatar a existência de diferenças estatisticamente significativas entre as crianças com câncer e as com as outras doenças, sendo que as primeiras apresentam um diagnóstico mais recente e deslocam-se ao hospital com maior frequência. Esses dados poderão estar relacionados com a severidade da doença, sendo que o câncer é responsável pelo maior número de mortes relacionadas com doenças na infância (Thompson \& Gustafson, 1996). Para além disso, o tratamento dessa doença, além de ser geralmente agressivo (Eiser, 1990), requer deslocamentos muito frequentes ao hospital, principalmente nas fases mais agudas (Thompson \& Gustafson, 1996). Com base nesse fato, é expectável que estas sejam as crianças que mais veem a sua rotina afetada, sendo nesse grupo encontrado o maior número de internamentos, faltas à escola e impedimentos na realização de atividades. Esses dados vão também ao encontro da bibliografia, sendo que o grande número de internações, a abstenção escolar e os tratamentos dolorosos e invasivos são reportados como fatores de estresses psicossociais particularmente presentes nas crianças com câncer (Moore, 2002).

No que se refere ao objetivo 1, foi possível concluir que apenas foram encontradas diferenças estatisticamente significativas na subescala "sintomas emocionais", sendo o grupo das crianças com câncer o que apresenta um valor mais elevado nessa escala. Os resultados encontrados contrariam diretamente o estudo de Siefert, Wittmann, Farquar, e Taisma (1992) que, ao comparar 
crianças com câncer e com asma, não encontrou diferenças estatisticamente significativas ao nível do ajustamento psicológico e social. Para além disso, esses dados contrariam a visão não categorial defendida por Pless e Pinkerton (1975), já que não são encontradas diferenças entre crianças com doença e crianças sem doença, mas entre crianças com doenças crônicas diferentes.

Os resultados podem estar associados à maior gravidade dessa doença e ao seu prognóstico, diante das outras duas doenças do estudo, sendo várias as pesquisas que relacionam esses fatores com o ajustamento psicológico (Lavigne \& Faier-Routman, 1992, apud Wallander \& Thompson, 1995; Zashikhina \& Hagglof, 2007). De acordo com Gortmaker (1985, apud Eiser, 1993), enquanto as crianças com asma e com doença renal têm uma esperança de vida normal, as crianças com câncer veem essa estimativa reduzida para os $60 \%$. Alguns estudos descritivos acerca de crianças com câncer parecem também suportar essa hipótese, já que têm demonstrado que esse grupo de crianças apresenta um maior risco de problemas comportamentais, principalmente de internalização (Thompson \& Gustafson, 1996).

A atribuição dos diferentes comportamentos à doença da criança foi também analisada neste estudo, o que se constitui como uma mais-valia na análise dos resultados, sendo que essa área tem sido frequentemente negligenciada na literatura (Bradford, 1997). A análise dessa questão permite-nos clarificar alguns dos resultados apresentados anteriormente, já que são justamente os pais das crianças com câncer os que atribuem um maior número de alterações comportamentais à doença. Se nos debruçarmos sobre as questões nas quais essas diferenças são significativas, podemos ainda constatar que a maioria se enquadra na subescala "sintomas emocionais". É possível então concluir que os pais dessas crianças consideram que a doença (câncer) é a principal causa para as alteraçóes no ajustamento da criança, nomeadamente ao nível dos seus sintomas emocionais (e.g., maior tristeza, receios e preocupações).

$\mathrm{Na}$ POS, foram encontradas diferenças ao nível do brincar social, sendo possível perceber que as crianças sem doença apresentam menos comportamentos de brincadeira em grupo com os pares. Num primeiro olhar, esses dados parecem contrariar a ideia defendida por Meijer e Sinnema (2000), que postulam que as crianças com doença tendem a ter mais dificuldades na interação com os pares. No entanto é fundamental ter em conta o contexto em que esses comportamentos ocorrem: ambiente hospitalar. Tendo em conta que as crianças com doença crônica se deslocam com maior frequência ao hospital, esse contexto é-lhes mais familiar, podendo sentir-se mais confortáveis para interagir com seus pares. Em alguns casos, é ainda possível supor que algumas crianças com doença se encontram com regularidade nesses espaços, criando laços entre si. 
No que se refere à escolha dos brinquedos, os resultados vão ao encontro do sugerido na literatura: quando as crianças estão ansiosas ou sob estresse, preferem brincar com brinquedos que estejam relacionados com a causa da sua ansiedade (Gariepy \& Howe, 2003).

Como foi referido na revisão bibliográfica, não existe uma relação linear entre o ajustamento psicológico das crianças e a presença de uma doença crônica. Para além disso, a comparação entre crianças com doenças crônicas diferentes é ainda reduzida, sendo encontrados resultados pouco consistentes. Neste estudo, foram encontradas algumas diferenças significativas nas crianças com câncer em comparação com os outros grupos, quando o ajustamento psicológico é medido por um questionário aplicado aos pais. No entanto a observação direta do brincar das crianças não corrobora essas diferenças, sendo que a única diferença entre os grupos se centra nas crianças sem doença (grupo que apresenta menos comportamentos de brincar com os pares). Dessa forma, é possível constatar que a perspectiva dos pais acerca do ajustamento dos filhos nem sempre é coincidente com a avaliação deste mesmo ajustamento mediante a observação direta do comportamento da criança. Esses resultados vão ao encontro do sugerido por alguns autores relativamente ao fato de ser importante englobar as crianças nos estudos, já que o relato dos pais apenas nos permite aceder a um lado da realidade (Eiser, 1993; Thompson \& Gustafson, 1996).

Relativamente ao objetivo 2, é possível concluir que quanto mais recente é o diagnóstico, mais elevado o valor na subescala de sintomas emocionais. Esse resultado não vai no mesmo sentido da literatura, sendo que, no estudo de Zashikhina e Hagglof (2007), não foi encontrada nenhuma relação entre a duração da doença e a saúde mental das crianças. Thompson e Gustafson (1996) referem ainda que essa é uma variável pouco estudada na área do ajustamento, fazendo referência a alguns estudos que relacionam uma maior duração da doença com maiores problemas de ajustamento. No que se refere à periodicidade com que a criança se desloca ao hospital, foi também possível perceber que quanto maior essa periodicidade, maiores os problemas emocionais. Esses resultados contrariam o estudo de Bradford (1997), no qual não foram encontradas associaçôes significativas entre a saúde objetiva da criança (na qual se englobava, entre outros, a frequência com que estas tinham de se deslocar ao hospital) e o ajustamento. No entanto é fundamental ter em conta que, neste estudo, apenas eram alvo de investigação crianças com doenças do fígado.

Relativamente à medicação, os resultados indicam que as crianças em tratamento de quimioterapia apresentam mais sintomas emocionais do que as que apenas tomam medicação por comprimidos via oral. Isso vai ao encontro do sugerido por Barros (2003), já que a quimioterapia é um tipo de tratamento 
perturbador das atividades diárias, sendo, por isso, mais difícil de gerir.

Foi ainda possível concluir que as crianças que são impedidas de realizar alguma brincadeira apresentam valores mais elevados ao nível dos sintomas emocionais. Como referido anteriormente, a possibilidade de uma criança realizar atividades normativas e lúdicas é fundamental para o seu desenvolvimento (Landreth, 2002; McCue, 1988). Dessa forma, os resultados encontrados vão ao encontro do sugerido pela literatura, já que as crianças, ao serem impedidas de realizar algumas atividades diárias e ao verem restringidas as suas brincadeiras, apresentam maiores problemas em termos emocionais. No entanto não foram encontradas diferenças estatisticamente significativas relacionadas com o internamento ou com as faltas à escola, o que deve também ser analisado. Tal como mencionado na revisão bibliográfica, no estudo de Stein e Jessop (1984) também não foi encontrada nenhuma relação entre o ajustamento e o número de hospitalizações, embora tenha sido encontrada uma relação moderada entre este e a abstenção escolar.

Relativamente à escala de observação do brincar, os resultados indicam que as crianças que são impedidas de realizar algumas brincadeiras apresentam um menor número de comportamentos de brincar em grupo. Esses dados vão ao encontro do sugerido por Meijer e Sinnema (2000), que defendem que a imposição de restrições na realização de atividades diárias pode desencadear dificuldades ao nível da interação social com os pares.

No que se refere à percepção dos pais acerca do impacto da doença no ajustamento da criança, é possível constatar que é novamente na subescala sintomas emocionais do SDQ que se verifica um maior número de correlações, objetivo 3. Dessa forma, quanto mais severas são as consequências percebidas pelos pais, maior o valor nessa subescala. Para além disso, quanto maior a percepção de controle por parte da criança, mais sintomas emocionais são identificados. Os valores nessa subescala são também mais elevados quanto menor a compreensão e a coerência atribuída à doença. Foi ainda encontrada uma correlação noutra subescala do questionário de capacidades e dificuldades, sendo que quanto maior a percepção de uma duração cíclica da doença, maior o valor na subescala de hiperatividade.

A análise da regressão linear múltipla permite constatar que a subescala de sintomas emocionais se relaciona tanto com variáveis associadas à saúde objetiva (periodicidade) como com alguns itens do IPQ-R (saúde subjetiva). Esses dados diferem dos encontrados no estudo de Bradford (1997), no qual se verifica que a percepção das mães acerca da doença é um melhor preditor de seu ajustamento do que a sua saúde objetiva. 
Relativamente à escala de observação do brincar, os resultados indicam que quanto maior a percepção dos pais acerca da eficácia do tratamento, maior a frequência de comportamentos de "brincar". Esses resultados vão ao encontro do postulado por Perrin, MacLean Júnior, e Perrin (1989), já que sugerem que as percepções dos pais têm impacte na própria criança e na forma como esta lida com a doença. Dessa forma, as crianças cujos pais percepcionam uma maior eficácia do tratamento parecem estar mais disponíveis para brincar, indicando um melhor ajustamento psicológico.

\subsection{Limitações}

É importante fazer referência a algumas limitações deste estudo, que exigem alguma precaução na interpretação e generalização dos resultados. Em primeiro lugar, de forma a conseguir uma amostra significativa, foi necessário abranger crianças com idades muito distintas, o que é uma limitação frequente em estudos que se debruçam sobre crianças com doença crônica (Eiser, 1993).

Dado o local e o ambiente onde os questionários foram aplicados, foi feito um esforço por selecionar instrumentos curtos e fáceis de responder, de modo a importunar ao mínimo os pais das crianças. A necessidade de criar um questionário de preenchimento simples e rápido limitou ainda o número de variáveis a ter em conta no estudo. Dessa forma, segundo os modelos mais atuais do ajustamento psicológico, na análise desta variável, é fundamental ter em conta não apenas as características da doença, mas também as características da própria criança e do seu meio. Isso não foi possível neste estudo, sendo que se sugere que essas variáveis sejam tidas em conta em futuras investigaçôes.

No que se refere à aplicação da POS, é importante ter em conta o fato de essa aplicação ter de ser feita em contexto naturalista. A ausência de gravações impediu assim a cotação da escala por mais do que uma pessoa, além de não possibilitar a análise dos temas das brincadeiras.

Por último, importa ainda analisar a seleção das doenças do estudo. Algumas dessas doenças, tal como a doença renal crônica, são frequentemente encaminhadas para outros hospitais mais especializados, o que faz com que o número de crianças com doença renal crônica alvo de tratamento no Hospital Infante D. Pedro seja muito reduzido. Por isso, foram inclusas no estudo crianças com doenças do foro uronefrológico que podem desencadear uma doença renal crônica, mas que podem não ser ainda consideradas doenças crônicas. Em futuras investigações, poderia ser também interessante acrescentar outras doenças que têm um grande impacte no dia a dia das crianças, tais como a diabetes, bem como doenças crônicas que envolvam comprometimento cerebral. 


\section{CONCLUSÃO}

Ao longo dos anos, a doença crônica infantil tem sido alvo de grande curiosidade e fomento para a investigação científica. Em nível médico, tem sido possível melhorar os tratamentos dessas crianças, aumentando assim a sua esperança média de vida. No entanto, tal como referido anteriormente, é fundamental não descurar a vertente psicológica na gestão dessas doenças. Por isso é atualmente possível encontrar um largo leque de estudos que se centram nas questões do ajustamento. Alguns autores optam por uma visão restrita do conceito de ajustamento, definindo-o como adaptação à doença, o que impede a comparação entre crianças saudáveis e aquelas com doença crônica. Por outro lado, a maioria das investigaçôes que se centram na definição mais alargada de ajustamento, enquanto medida do funcionamento da criança no seu dia-a-dia, acabam também por ser limitadas já que se centram apenas na comparação entre crianças saudáveis e crianças com uma doença crônica específica (existindo doenças que têm recebido comparativamente mais atenção do que outras).

Este estudo procurou assim colmatar uma das principais lacunas nesta área, permitindo-nos averiguar não só a existência de diferenças entre crianças com doença crônica e crianças sem doença, mas também entre crianças com doenças crônicas diferentes. Para além disso, são englobadas neste estudo doenças com características e progressões muito distintas, algumas das quais menos abordadas nas investigações nesta área.

Tendo por base as comparações referentes ao ajustamento psicológico entre os diferentes grupos, foi possível encontrar diferenças estatisticamente significativas entre as crianças com câncer e os restantes grupos, contrariando assim a visão não categorial de Pless e Pinkerton (1975), já que as diferenças não se devem à presença ou ausência de doença crônica, centrando-se numa doença específica. A análise das características específicas das doenças permitiu ainda identificar algumas variáveis que parecem ter impacte no ajustamento emocional, nomeadamente o tempo de doença, a periodicidade com que a criança se desloca ao hospital e a impossibilidade de realização de atividades lúdicas. Os resultados encontrados ao longo deste estudo parecem, assim, ir ao encontro da visão parcial ou modificada sugerida por Thompson e Gustafson (1996), no que se refere ao estudo das doenças crônicas e do ajustamento psicológico na infância. Dessa forma, os dados parecem corroborar a existência de condições que são partilhadas por todas as crianças com doença crônica, embora existam aspetos que parecem ser específicos de determinadas doenças.

Esta investigação reforça, assim, a ideia defendida atualmente na literatura, de que não existe uma relação linear entre o ajustamento e a doença crônica 
infantil. Dessa forma, foi possível perceber que a doença crônica não desencadeia necessariamente problemas de ajustamento, sendo que algumas crianças gerem esse acontecimento de forma adaptativa.

A utilização da observação do brincar como forma de avaliação do ajustamento da criança é também uma mais-valia desta investigação. Dessa forma, sendo o brincar um meio de expressão fundamental na infância, sua observação permitiu uma nova abordagem do ajustamento da criança, que não apenas a percepção dos pais acerca deste. Assim é importante sublinhar que, quando nos focamos na análise da Escala de Observação do Brincar, a única diferença encontrada quando se comparam os quatro grupos de crianças relaciona-se com o fato de as crianças sem doença apresentarem menos comportamentos de brincar em grupo com os pares do que as crianças com doença crônica. Esses resultados permitem, assim, perceber que a avaliação do ajustamento da criança pela observação direta do brincar nem sempre é coincidente com a perspectiva dos pais acerca desse ajustamento. Os dados sugerem também que a própria percepção dos pais acerca da doença e do tratamento tem impacte na forma como a criança gera esse acontecimento.

Espera-se, dessa forma, que este estudo dê um contributo importante, não somente na área da investigação acerca do ajustamento psicológico, mas também para fornecer orientações para a prática em nível hospitalar, permitindo uma melhor integração entre o apoio psicológico e os serviços médicos. 


\section{REFERENNCIAS}

Antão, J. (2013). O ajustamento de crianças com doença crônica e o brincar no hospital. (Tese de Doutorado), Universidade de Aveiro, Aveiro.

Barros, L. (2003). Psicologia pediátrica: perspectiva desenvolvimentalista (2a ed.). Lisboa: Climepsi.

Bradford, R. (1997). Children, families and chronic disease: psychological models and methods of care. London: Routledge.

Eiser, C. (1990). Chronic childhood disease: an introduction to psychological theory and research. Cambridge: Cambridge University Press.

Eiser, C. (1993). Growing up with a chronic disease: the impact on children and their families. London; Philadelphia: J. Kingsley Publishers.

Gariepy, N. \& Howe, N. (2003). The therapeutic power of play: examining the play of young children with leukaemia. Child: Care, Health \& Development, 29(6), 523-537.

Landreth, G. L. (2002). Play therapy: the art of the relationship (2a ed.). New York: Brunner-Routledge.

Lavigne, J. V. \& Faier-Routman, J. (1992). Psychological adjustment to pediatric physical disorders: a meta-analytic review. Journal of Pediatric Psychology, 17(2), 133-157.

McCue, K. (1988). Medical play: an expanded perspective. Children's Health Care, 16(3), 157-161.

Meijer, S. \& Sinnema, G. (2000). Social functioning in children with a chronic illness. Journal of Child Psychology \& Psychiatry \& Allied Disciplines, 41(3), 309-317.

Moore, I. (2002). Cancer in children. In L. L. Hayman, M. M. Mahon, \& J. R. Turner (Eds.), Chronic illness in children: an evidence based approach. (pp. 80103). New York: Springer.

Parten, M. (1932). Social participation among pre-school children. The Journal of Abnormal and Social Psychology, 27(3), 243-269.

Perrin, J. M., MacLean Júnior., W. E. \& Perrin, E. C. (1989). Parental perceptions of health status and psychologic adjustment of children with asthma. Pediatrics, 


$$
\text { 83(1), 26-30. }
$$

Piaget, J. (2010). Play, dreams, and imitation in childhood. New York: Norton.

Pless, I. B. \& Pinkerton, P. (1975). Chronic childhood disorder: promoting patterns of adjustment. London: Kimpton.

Rubin, K. (2001). The play observation scale (POS). Maryland: University of Maryland.

Santos, C., Pais-Ribeiro, J., \& Lopes, C. (2003). Adaptação e validação do "Revised Illness Perception Questionnaire" (IPQ-R) em doentes oncológicos. Arquivos de medicina, 17(4), 136-147.

Siefert, K., Wittmann, D., Farquar, S., \& Taisma, F. (1992). Similarities and differences between children with asthma and children with cancer: implications for preventive intervention. The Journal of Primary Prevention, 13(2), 149-159.

Stein, R. \& Jessop, D. J. (1984). Relationship between health status and psychological adjustment among children with chronic conditions. Pediatrics, 73(2), 169-174.

Thompson, R. J. \& Gustafson, K. E. (1996). Adaptation to chronic childhood illness. Washington: American Psychological Association.

Wallander, J. L. \& Thompson, R. J. (1995). Psychosocial adjustment of children with chronic physical conditions. In M. Roberts (Ed.), Handbook of pediatric psychology (2a ed., pp. 124-141). New York: Guilford.

Wallander, J. L. \& Varni, J. W. (1998). Effects of pediatric chronic physical disorders on child and family adjustment. Journal of Child Psychology \& Psychiatry \& Allied Disciplines, 39(1), 29-46.

Weiland, S. K., Pless, I. B., \& Roghmann, K. J. (1992). Chronic illness and mental health problems in pediatric practice: results from a survey of primary care providers. Pediatrics, 89(3), 445-449.

Zashikhina, A. \& Hagglof, B. (2007). Mental health in adolescents with chronic physical illness versus controls in Northern Russia. Acta Paediatrica, 96(6), 890-896. 